\title{
Homogeneous Gelation Leads to Nanowire Forests in the Transition Between Electrospray and Electrospinning
}

\author{
Lin Lei, Shensheng Chen, Catherine J. Nachtigal, Tyler F. Moy, Xin Yong, Jonathan P. Singer* \\ *Corresponding Author: jonathan.singer@ rutgers.edu
}

\section{Introduction}

There are several distinct communities in the field of electrostatic deposition. Electrostatic corona spray has been a standard tool for automotive, pharmaceutical, agricultural, and other commercial paint applications. Filament-based approaches in the far field (i.e. electrospinning) have recently attained industrial applications for making nanofiber mats as filters and scaffolds, ${ }^{1}$ while the near field methods (i.e. electrohydrodynamic printing) enables direct writing of polymer or nanostructured filaments. Electrospray, despite being the first method developed in electrostatic deposition, ${ }^{2}$ has been primarily employed in metrology through mass ionization. ${ }^{3}$ Recently, however, electrospray deposition (ESD) has gained increasing attention in nanotechnology and biomedical applications with its unique ability to deliver nanograms of material per droplet. ESD has been utilized for, among other applications, nanostructured polymer coatings, the delivery of cells and other bioactive media, and the synthesis of hierarchical functional oxides. ${ }^{4}{ }^{5}$ Even being restricted to dissolved solutions (e.g. polymer or other small molecule processing), the electrostatic deposition exhibits a breakdown of deposit morphologies into "wires", ${ }^{6-9}$ "particles," , 7, 9-15 and "films," 16 with the wires (produced by filament-based approaches) lying flat on a substrate, the particles (produced by electrostatic corona spray or ESD) forming hierarchical coatings, and the films, which can arise from any of the methods, covering the entire target region or dewetting into localized regions. A brief collection of examples, with a few lesser-observed morphologies, can be found in Table S1.

The deciding factor in the formation of these morphologies is the dynamic viscosity of the solution during the process. If the initial viscosity is high and increases during the evaporation of the carrier solvent, droplets do not form and instead the solution spins as a filament. With the whipping of the electrostatic spinning process, this regime produces mats of in-plane nanowires (NWs). If the viscosity begins low and then increases rapidly, a particulate spray is formed. If the viscosity does not appreciably increase during the deposition process, a film is formed in a wetting regime. Intermediate regimes of processing lead to other desirable (or undesirable) effects. For example, a certain degree of film-forming tendency in wire or particulate sprays can lead to solvent-welding of the mats or particles into bicontinuous films. This is advantageous for improving the mechanical robustness of the deposits and promoting resistance to the corona pitting, which is a notable failure mode of powder electrostatic sprays. ${ }^{17}$ Despite this, one surprising observation is that there are no reports of ESD of NW forests, which would be a natural intermediate regime between wire and particulate depositions. The forest morphology is characterized by preferential alignment of short NWs along the direction of the field and can be interpreted as an intermediate of particulate and NW mat morphologies, since the filamentation would naturally be expected to occur in the direction of the driving force. This is analogous to the near-field electrohydrodynamic printing, where the high field pulls what would be an electrospun filament directly to the substrate before the far-field whipping that creates mats initiates. ${ }^{18}$ All that should be necessary for this to occur is for the viscosity of a sprayed droplet in ESD to become great enough when the droplet approaches the substrate so that each droplet presents a similar phenomenological arrangement of the printing nozzle (Figure 1a). 


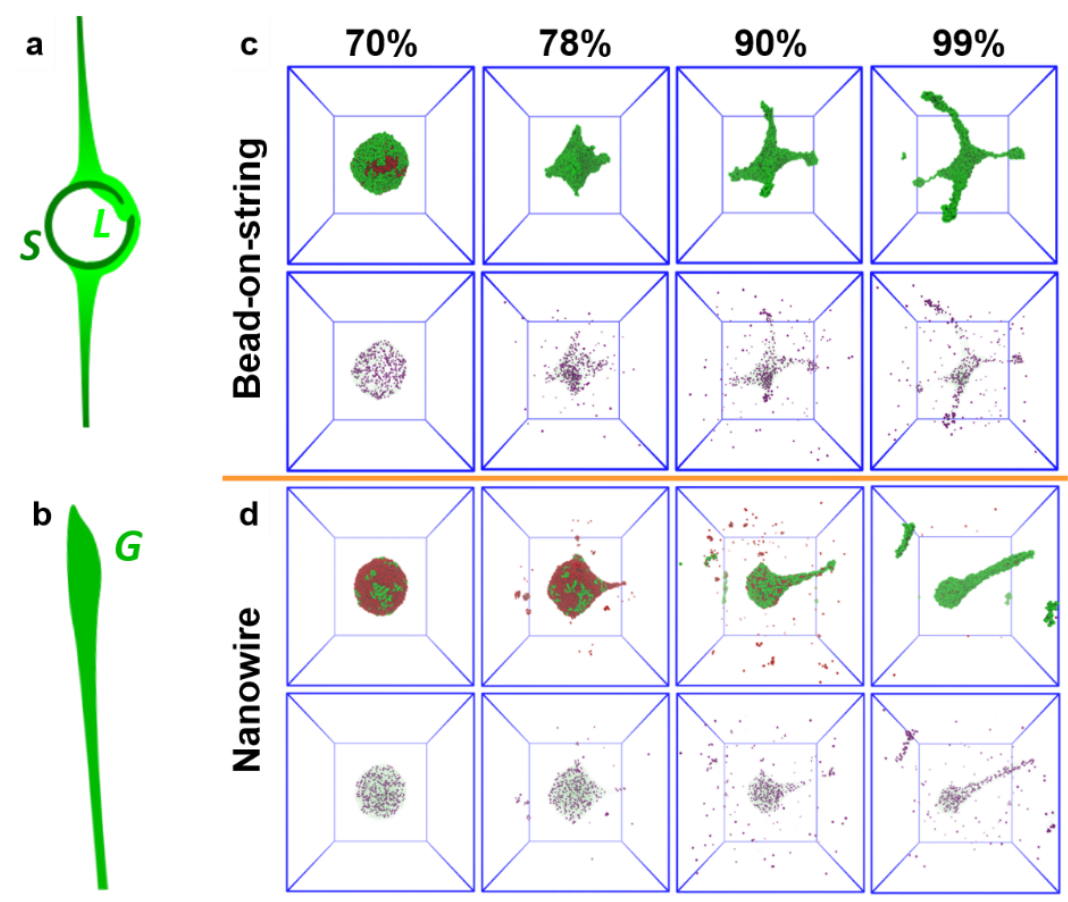

Figure 1: Formation of bead-on-string and nanowire morphologies in electrospray deposition. (a) Schematic of the proposed bead-on-string formation mechanism where the immobile solid-like ("S", dark green) exterior of the droplet is unable to participate in the fission, while the more-liquid interior ("L", light-green) is able to escape to the surface to participate in fission. (b) Schematic of the formation of a nanowire where the intermediate viscosity gel ("G", green) is extruded into an asymmetric filament. (c,d) Dynamic evolution of droplet morphology and ion distribution in the DPD simulations under the (c) physical evaporation (100 beads per time step) and (d) homogeneous removal of solvent bead ( 2 beads per time step), which shows the development of the bead-on-string and nanowire geometries respectively. The number denoting each column is the percentage of removed solvent beads, representing different times in the electrospray transit. The green and red spheres in the upper panel are polymer and solvent beads. The magenta spheres in the bottom panel are charged ion beads while the light green dots in the background represents both polymer and solvent. Vapor beads are not displayed for clarity.

Certainly, the viscosity of evaporating droplets increases during ESD and there exist transition behaviors. The most common and intriguing observation in our studies at the transition between ESD and electrospinning is a "bead-on-string" geometry (Figure 1a), though Almería and Gomez also reported small ring particles when electrospinning is conducted near the breakdown of the filament. ${ }^{19}$ In the "bead-onstring" mechanism, the poles of the electrostatically deformed droplet will each produce a filament, while leaving some intact central mass. These filaments are a natural evolution of the Coulomb fission process, where an ESD droplet, upon reaching the Rayleigh limit of surface charge under rapid solvent evaporation, will create additional child droplets through the creation of Taylor cone pseudopods. ${ }^{20-22}$ Should Coulomb fission occur in a more viscous droplet, two filaments are generated instead. Since the filaments are composed of the most fluidic parts of the droplet, which have the ability to migrate to the poles, it can be expected the less-mobile parts are retained in the central mass. The reason there are such low-mobility central regions in the first place is the fact that evaporation is an interfacial process, in which the solvent is removed only from the surface of the droplet. This naturally creates a less-mobile skin of solute over the outer region of the droplet. Even if this skin is mobile enough to participate in the filamentation, there are 
doubtless other portions of the interior droplet that can more rapidly migrate to the forming Taylor cone, leaving it behind to solidify as the parent droplet. As a result, the formation of filaments never employs the entirety of a droplet: droplets either produce other droplets and then solidify or produce filaments only at their poles. This can also be viewed via the electrostatic capillary number of the droplet during evaporation. If the evaporation halts when the $\mathrm{Ca}$ is high for the whole droplet, there will likely be particles or shells as the final morphology. Alternatively, if the $\mathrm{Ca}$ is low for the interior of the droplet, but high for the shell, a bead-on-string morphology will form. This behavior has been previously observed by Merrill et al., labeled as "comet" particle. ${ }^{23}$ In this report, they also observed some elongated particles, but were only a small fraction of the population. Zhao et al. also reported some population of "nanopillars" at specific spray distances in photovoltaic polymers that show shear-oriented crystallinity. ${ }^{24}$

If the proposed mechanism is correct, what would be required to produce a NW forest is a rapid increase in viscosity of the entire droplet near a fission event. This is an atypical behavior for an in-flight droplet evaporating under substantial forced convection. Further, this increase would most likely have to occur in the timescale of a typical electrospray transit, which is $O(100 \mathrm{~ms})$. This is uncharacteristically fast for a majority of kinetic processes in solution that often progress by nucleation or diffusive processes. One transformation that could potentially satisfy these requirements is the homogenous formation of a gel, such as through a spinodal or other mechanisms. To induce the formation of NWs, the gelation time would have to be competitive with the evaporation timescale.

To test this hypothesis, we approached the problem using coarse-grained computational modeling of a model system and methylcellulose (MC) ESD experiments. The goal of the simulations was to establish that if homogenous viscosity transitions did occur, they would be associated with the tendency to form singular NWs, while heterogenous viscosity transitions would lead to shells, particles, or beads-on-strings. The experiments then sought to establish that the ESD of MC solutions in water:ethanol mixtures could satisfy the required kinetics.

\section{Simulation Results and Discussion}

Central to the morphology development in ESD is the interplay between electrohydrodynamics and evaporation of polymer solution droplets. We conducted electrostatic dissipative particle dynamics (DPD) simulations to uncover the full dynamic evolution of electrified droplets in flight. The model system was a charged droplet containing $10 \%$ polymer chain beads and $90 \%$ solvent beads with charge beads distributed evenly in both phases. Evaporation was modeled by removing solvent beads locally at the droplet surface. The rate of evaporation can be controlled by the number of beads removed per time step. This approach mimics the physical mass transport at the liquid-vapor interface and reproduces the $\mathrm{D}^{2}$ law as shown in Figure $\mathrm{S} 1 .^{25}$ The initial charge density of the droplet was small so that a stable spherical droplet could be obtained. Upon solvent evaporation, the charge density increases and eventually drives the deformation and fission of the droplet.

Figure 1c shows the representative morphologies of a polymeric droplet during a Coulomb fission event. As the charge density reached the Rayleigh limit, multiple Taylor cone pseudopods were formed at the surface of droplet (Supplementary Video 1). Ion beads were emitted from the pseudopods into the vapor phase, followed by the protrusion of polymer chains from the central mass. Due to the strong entanglements of polymer chains, the capillary rupture of the filaments was inhibited. Instead the central region of the filaments underwent a necking process while the tips formed bulges. These orchestrated events lead to the bead-on-string morphology. To provide insight into the solute distribution inside the droplet, we characterized the polymer density profile in an uncharged droplet under evaporation. Figure 2a confirms the development of a densified polymer skin at the surface of droplet, which was driven by the evaporation- 
induced advection. This skin with much higher polymer concentration will naturally generate a pronounced viscosity gradient in the droplet. This result is consistent with the shell formation in a majority of sprays and also provides evidence that the bead-on-string morphology is associated with the presence of evaporation-driven viscosity gradient. Figure S2a-c compares the final morphologies of dried polymer in simulations performed at different evaporation rates, in which the skin formation was also confirmed (Figure S3). Consistent bead-on-string geometries were obtained.
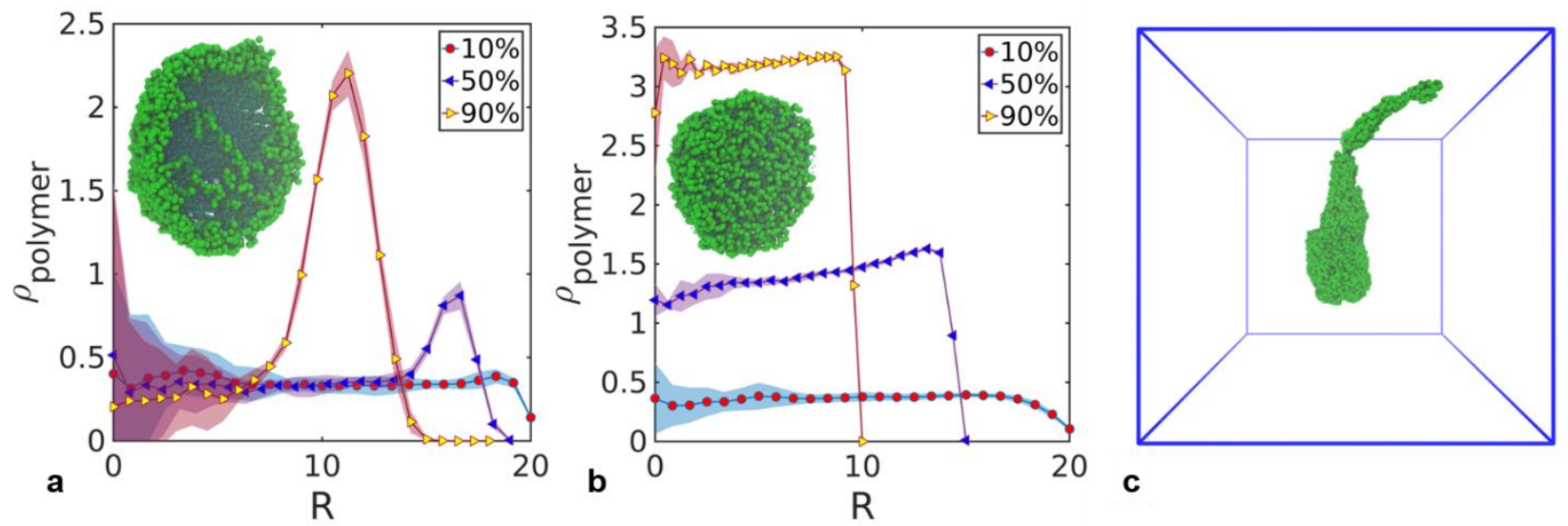

Figure 2: Evolution of polymer density distribution during evaporation. (a,b) Radial polymer density profiles in the uncharged polymeric droplets in the DPD simulations at the instance when $10 \%, 50 \%$, and 90\% of solvent has been evaporated. The evaporation rates are (a) 100 and (b) 1 bead(s) per time step. The shaded error band represents the standard deviation of 5 independent runs. The insets are the corresponding cross-sectional snapshots of the droplet after evaporation of $90 \%$ of solvent. (c) Final morphology of the charged polymeric droplet obtained at the evaporation rate of 1 bead per time step.

The desired gelation of a polymer network would rapidly and uniformly increase the droplet viscosity, which was not captured in the simulations above. To model this regime of ESD, we performed another set of simulations by removing randomly selected solvent beads from the droplet, not limited to the surface. This approach guarantees a homogeneous distribution of polymer in the entire droplet during the morphology development. Figure 1d demonstrates that the multiple protrusions of polymer filaments under Coulomb fission were strongly suppressed. Only one filament was observed in the simulation, resulting in a tadpole-shaped droplet (Supplementary Video 2). As this filament further elongated and grew, a nanowire was formed eventually. Additional simulations at different rates of removing the solvent beads shown in Figure S2d-f indicates the formation of nanowire is not sensitive to the simulation conditions given that the droplet viscosity increases uniformly.

In experiments, the physical evaporation rate is typically much higher than the rates of mutual diffusion of polymer and solvent in the droplet. However, the evaporation rate is readily adjustable in the DPD simulation. This allows us to uniquely predict what will happen if the characteristic time scales of evaporation and diffusive processes are comparable. Figure $2 b$ shows that polymer maintained its homogeneous distribution inside the droplet when evaporation was simulated at a much lower rate (e.g., 1 bead per time step). No skin formation was observed under slow evaporation. In this regime, the formation of the Taylor cone pseudopods was inhibited, and the droplet only developed one filamentous protrusion during deformation, as shown in Supplementary Video 3. The final geometry was nanowire (Figure 2c). The entire morphology development resembles the one observed in Supplementary Video 2 where the 
solvent was uniformly removed. These simulations provide strong evidence that the nanowire formation requires uniform droplet viscosity while the presence of significant viscosity gradient promotes the development of bead-on-string morphology.

\section{Electrospray Results and Discussion}

Moving to the experimental system, MC possesses a lower critical solution temperature (LCST) in water and water:ethanol blends, though the ethanol is predicted to rapidly evaporate. Above the LCST, the MC and water phases separate and gel. The mechanism for this separation and gelation has been an active topic of research. Recent work, much of it conducted by Bates and Lodge, suggests that the formation of MC gels is mediated by fibrils that form from aggregates that assemble in the low temperature solution. ${ }^{26-28}$ Also relevant to the ESD process is that, once formed, the fibril network is shear thickening ${ }^{29}$ and highly viscous in extension. ${ }^{30}$ Prior kinetic investigations have shown three characteristic time scales for MC gelation, ${ }^{31}$ with the most rapid potentially satisfying the required kinetics.

MC solutions were sprayed at room temperature that would place them in a single liquid phase. Initially, it was expected that the spray target would need to be above the LCST $\left(\sim 40{ }^{\circ} \mathrm{C}\right.$ at $\left.1 \%\right)$ to obtain the gelation and NW formation, and Figure 3 shows characteristic SEM images from MC conducted at different substrate temperatures and flow rates. At room temperature, there is some greater tendency to form agglomerated structures; however, the NW formation is remarkably robust at all temperatures. This is notable because we were unable to obtain NWs in poly(N-isopropylacrylamide) (PNIPAAm, Figure S4a), another LCST, but non-gelling polymer, even at high temperature that may be expected to trigger a spinodal. The same was true of gelatin that forms strong gels through a different, UCST, formation mechanism (Figure S4b), which only showed electrospinning and bead-on-string morphologies in all conditions tested. Wires were obtained in hydroxypropyl methylcellulose (HPMC), another LCST cellulose ether that forms weak gels (Figure S4c). It is therefore likely that both the gelation and the fibrillation mechanism that distinguishes cellulose ethers from other polymers is the origin of this difference. This said, the thermodynamic studies of fibrillation have shown this mechanism to require timescales of hours. ${ }^{26}$ However, the ESD process is highly non-equilibrium and possesses three distinct characteristics: ethanol, evaporation, extreme shear/extension rate, and high surface charge. Ethanol is rapidly evaporating in these mixtures and has been known to suppress gelation, ${ }^{32}$ so is likely not the cause. Evaporation will increase the concentration of the sprayed solution; however, were this the key effect, bead-on-string morphologies would likely be observed as with other evaporation-dominated results. Ionic effects are known to have a large influence on the gelation of polymers, for example, the addition of salts to either lower or raise the LCST of MC. ${ }^{33} \mathrm{pH}$ is also known to have an effect on gelation kinetics. ${ }^{34}$ It is reasonable to expect that free surface charge may also alter the gelation behavior and kinetics. To estimate the magnitude of the strain rate, we can consider that Gomez and Tang verified through high-speed photography that a fission event occurs in $<1 \mu \mathrm{s}$, which places the strain rate conservatively at $\dot{\gamma}=10^{6} \sim 10^{7}$, which may induce order in the nematic-like fibrils. Certainly, both of these effects, charge and strain, are also at play in electrospinning. Cellulose ethers, primarily HPMC, have been deposited by electrospinning previously; however reports of MC electrospun mats are limited and indicate that the mats are unusually fused ${ }^{35}$ in a very similar fashion to the room temperature results reported here. This suggests that the shear/charge-free state of the filaments on the substrate after deposition are more fluid-like. While these are only speculative indications of a possible mechanism, the fact remains that MC and HPMC can form NWs, which, according to the simulation results, indicates a transition in viscosity at a more rapid rate than solvent evaporation. This established, we now evaluate how modifications in the electrospray parameters can be used to alter or disrupt the morphology of the NWs and their forests. It would be expected that this behavior would be very sensitive to changes in the material viscosity and surface charge, and we approached this through changes 
in (1) flowrate, (2) molecular weight, (3) concentration, and (4) additive content. Effects were determined by examining short-time sprays of isolated single wires (Figure S5-7). Extracted parameters are shown in Figure 4, with a notional aspect ratio (AR) defined as the ratio of the mean length and mean diameter of the NWs. While this definition captures some behaviors, it is sensitive to the production of child droplets, that have an oversized effect in reducing the AR considering their low mass fraction of the sprayed polymer.
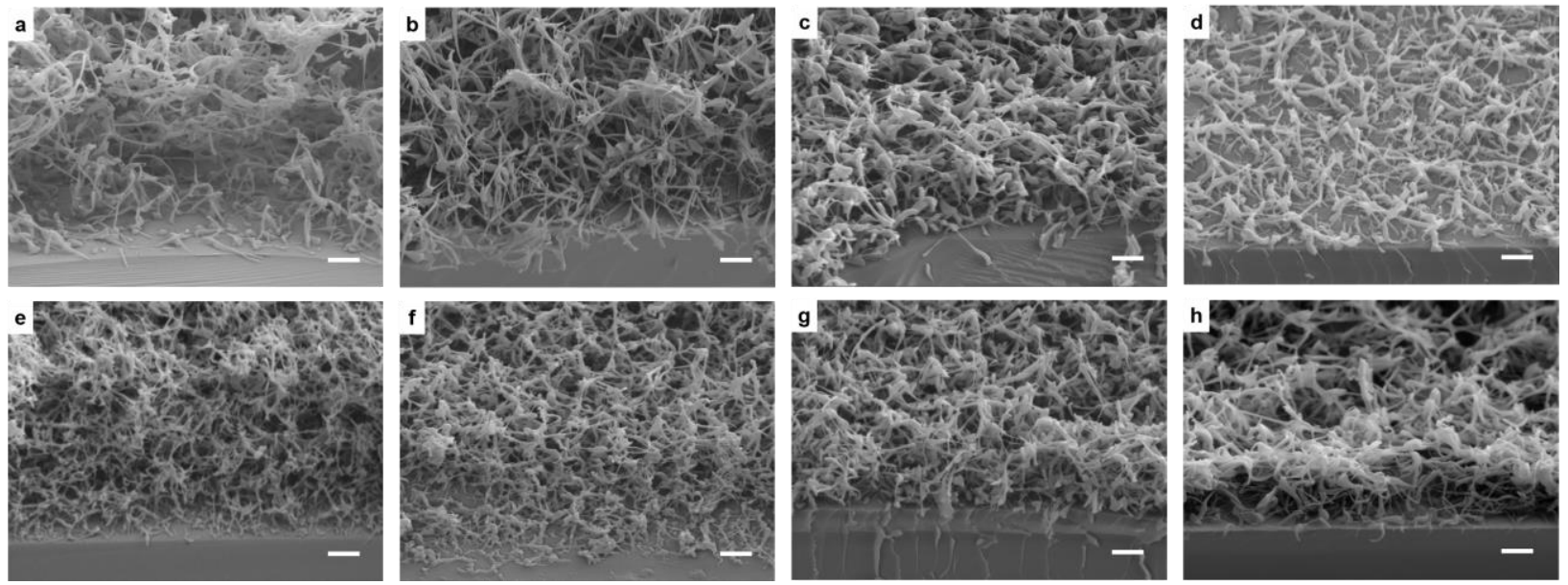

Figure 3. Parametric spray of MC nanowires. SEM images of $1 \mathrm{wt} \%, 14 \mathrm{kDa} \mathrm{MC}$ in 3:2 volume-basis water:ethanol blend sprayed with different substrate temperatures and different flow rates. (a) $30{ }^{\circ} \mathrm{C}$, (b) $40{ }^{\circ} \mathrm{C}$, (c) $50{ }^{\circ} \mathrm{C}$, (d) $110{ }^{\circ} \mathrm{C}$ at $0.25 \mathrm{~mL} / \mathrm{hr}$ for $30 \mathrm{~min}$; (e) $0.02 \mathrm{~mL} / \mathrm{hr}$, (f) $0.05 \mathrm{~mL} / \mathrm{hr},(\mathbf{g}) 0.15 \mathrm{~mL} / \mathrm{hr}$, (h) $0.25 \mathrm{~mL} / \mathrm{hr}$ of substrate temperature of $90{ }^{\circ} \mathrm{C}$ at a constant solids quantity of $1.25 \mathrm{mg}$. All sprays were conducted at a spray distance of $4 \mathrm{~cm}$. All scale bars are $1 \mu \mathrm{m}$.

The flow rate in ESD is known to alter the droplet size $a^{36}$ :

$$
d=\alpha\left(\frac{Q^{3} \varepsilon_{0} \rho}{\pi^{4} \sigma \gamma}\right)^{\frac{1}{6}}+d_{o}
$$

Where $\alpha$ is a constant which related to the fluid's dielectric permittivity, $\rho$ is the density, $\gamma$ is the surface tension, $\sigma$ is the electrical conductivity, $Q$ is the flow rate, $\varepsilon_{0}$ is the permittivity of free space and $\mathrm{d}_{\mathrm{o}}$ is a small droplet diameter only significant at low flow rates. This change in droplet size arises from the balance in surface charge and tension, and the amount of charge per droplet is also affected by flow rate proportionally to $Q^{-\frac{3}{4}}$. From forest morphologies in Figure 3, the reduction of diameter of the NWs is readily apparent, creating a more open foam-like structure. From the individual wire measurements, the reduction in both length and diameter with reducing flow rate is apparent, and within these trends, the effects of droplet size on evaporation and charge effects. At higher flow rates, the aspect ratio and mean wire dimensions appear to stabilize below the peak value at $0.1 \mathrm{~mL} / \mathrm{hr}$ due to the increased incidence of child droplets from the slowed evaporation of the larger droplets, which are able to emit child droplets from the forming filament for a longer period of their evolution. At the lowest flow rate of $0.02 \mathrm{~mL} / \mathrm{hr}$, mean AR is also greatly reduced despite many of the wires possessing similar ARs to the higher flow rates again because of the production of child droplets. This can be seen directly in the high asymmetry of the 0.02 $\mathrm{mL} / \mathrm{hr}$ box plot in Figure 4, extending into very long wires, but tightly focused around the smaller lengths. The increase in this population is attributed to the higher surface charge increasing the extrusion force on 
the smaller droplets. This explains why the foams in Figure 3 appear to be high AR in their filaments, as the child droplets decorate the foam rather than alter its structure.

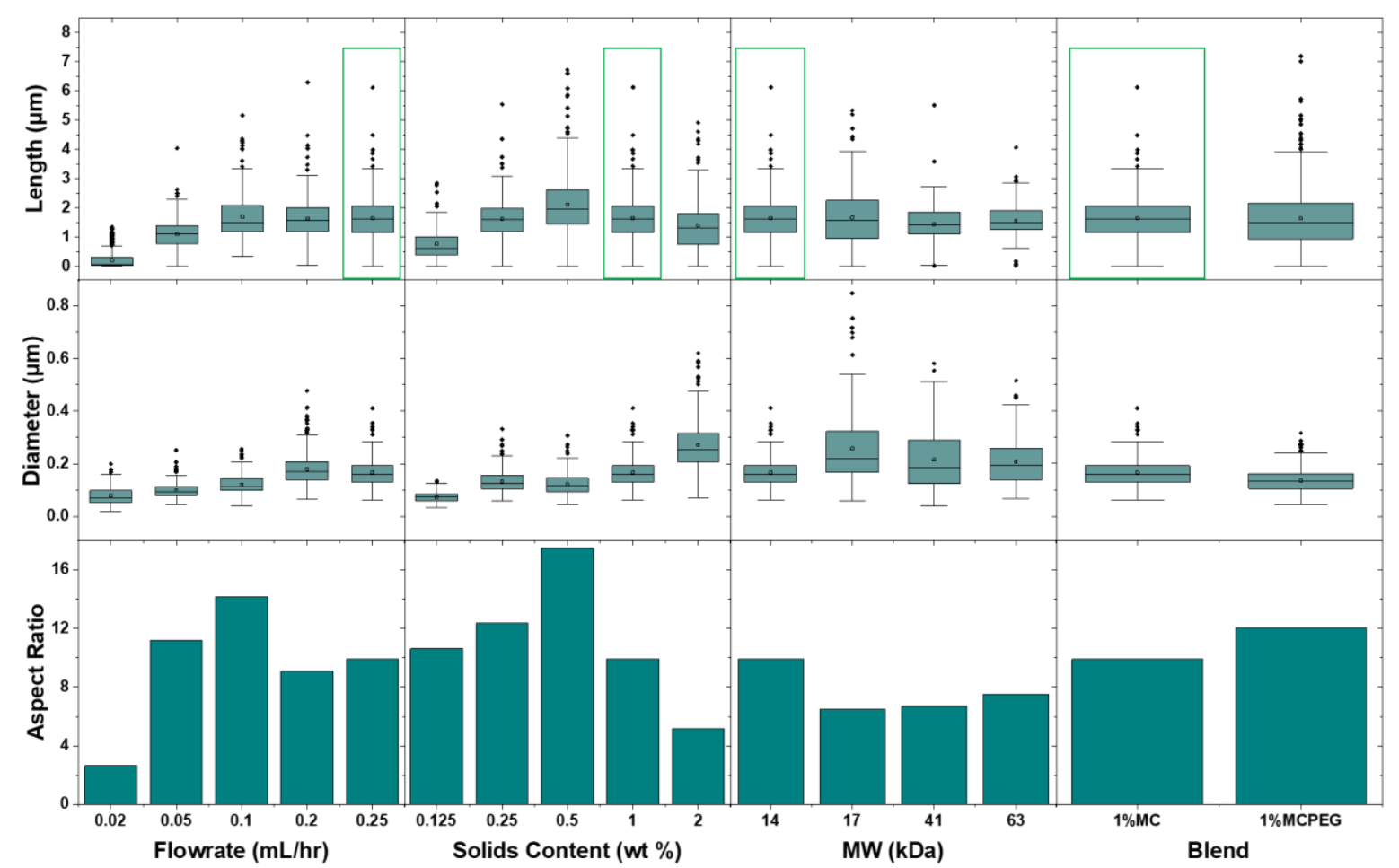

Figure 4: Parametric study of nanowire parameters from short-time sprays. Dependence of length (top), diameter (middle), and aspect ratio (bottom) of single wires on ESD parameters of MC films at different flowrates (left), solids content (left-middle), molecular weight (right-middle), and blending at a 5:1 ratio of MC:PEG 400. All sprays were conducted at a spray distance of $4 \mathrm{~cm}$ and substrate temperature of $90{ }^{\circ} \mathrm{C}$ from a 3:2 volume-basis water:ethanol blend. The green-boxed sample is the baseline for all sets ( $1 \mathrm{wt} \%$, $14 \mathrm{kDa} \mathrm{MC}, 0.25 \mathrm{~mL} / \mathrm{hr})$.

The concentration results also highlight this interplay between the evolution of a single wire and production of child droplets. Increasing the loading of solids can be viewed as setting a timer on the NW formation process. When the loading is high, droplets will quickly become too viscous to be extruded at a rapid rate, instead approaching bead-on-string morphologies as the surface evaporation outpaces the extrusion. Conversely, when the loading is low, the gel will be weak enough to allow for the emission of child droplets during the extrusion. The net result is the peak in AR seen in Figure 4 at $0.5 \mathrm{wt} \%$.

Molecular weight of MC has not been demonstrated to have a specific effect on the LCST temperature, ${ }^{26}$, ${ }^{37}$ however, the viscosity of gels has been previously linked to MW, with higher MW polymers leading to higher viscosity gels. ${ }^{26}$ The effects on the produced NWs is more subtle than the concentration within the range of MW studied, but a step up in the wire diameter can be seen in Figure 4 for all of the higher MW MCs examined, resulting in a drop in AR for all MWs higher than the lowest of $14 \mathrm{kDa}$.

We employed four different additives expected to have very different effects: (1) silica nanoparticles, (2) ethylene glycol, (3) polyethylene glycol, and (4) poly(vinylpyrrolidone)-capped gold nanoparticles. All additives were substitutional, meaning that the total solids content in the drop was fixed. Silica nanoparticles can be expected to uniformly increase the viscosity of the droplet as the solvent evaporates, leading 
eventually to complete jamming. As a result, nanowire formation is halted mid-generation as a "chicken tender" morphology (Figure 5a, with the full series shown in Figure S8). Ethylene glycol can be viewed as a plasticizer, lowering the viscosity of the gel and raising the gel temperature ${ }^{32}$ however, it is also volatile. This means that, much as with the evaporating droplets of the usual spray case, there will be a natural viscosity gradient from the outside to the inside of the droplet, resulting in beads-on-strings despite having an overall lower mean viscosity (Figure 5b, with the full series shown in Figure S9). Raising the MW of the ethylene glycol by switching to low MW polyethylene glycol (400 Da) removes the volatility. This creates longer, thinner wires at the same loading that previously resulted in bead-on-string morphologies, providing strong evidence that the presence of any viscosity gradient will prevent the nanowire formation (Figure 5c, with the full series shown in Figure S10). This was verified with single wire measurements shown in Figure 4; however, the apparent effect in the single wire was smaller than the effect observed in the lattice qualitatively. This suggests that the presence of the non-volatile plasticizer may allow for additional extrusion of the NWs created by the presence of the building electric field in the NW forest. To put it another way, the forest expands to lower the overall charge density and counter field developing on the target.
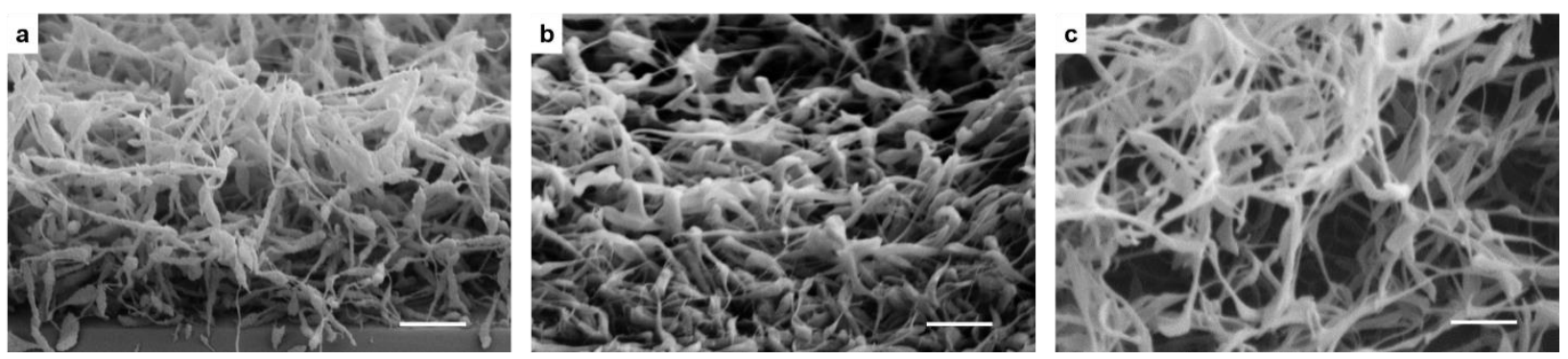

Figure 5: SEM images of $14 \mathrm{kDa}$ MC sprayed with different additives as $1 \mathrm{wt} \%$ from a 3:2 volume-basis water:ethanol blend in different mass ratios. (a) MC:silica particles (1:5) sprayed for 60min at substrate temperature of $90{ }^{\circ} \mathrm{C}$; (b) MC:EG (5:1) sprayed for 60 min at substrate temperature of $90{ }^{\circ} \mathrm{C}$; (c) MC:PEG $400(5: 1)$ sprayed $30 \mathrm{~min}$ at substrate temperature of $40^{\circ} \mathrm{C}$. All sprays were conducted at a spray distance of $4 \mathrm{~cm}$. All scale bars are $1 \mu \mathrm{m}$.

\section{Summary and Applications}

The ability to obtain a wide variety nanowire forests using low temperature, ambient condition spray processing at a highly scalable rate could be a huge benefit for continuous processes such as roll-to-roll manufacturing. In addition, these MC wires have the necessary properties to be a self-limiting electrospray deposition (SLED) as defined in our recent manuscript ${ }^{38}$ and confirmed by a hole depth array approach we have recently demonstrated. ${ }^{39}$ SLED enables the coating of complex 3D objects. As there are many methods for conversion structures of polymeric materials into other materials, by coating, pyrolysis, and sol gel methods, this work can be viewed as a starting point for the fabrication of a host of 1D architectures. Additionally, the MC NWs can "host" other materials, as shown with the gold nanoparticle results in Figure $6 a, b$. The goal of these experiments was, instead of to manipulate the viscosity of the forming wire, to demonstrate the ability to enhance the functionality of the deposit materials using these manipulations. In this case, the NW morphology prevents particle agglomeration and the resultant broadening of the singleparticle plasmon as shown in spectroscopic reflectometer experiments. It can be seen that two plasmon peaks emerge, characteristic of single particles $(\sim 545 \mathrm{~nm})$ and close-packed 1D particle chains $(\sim 650 \mathrm{~nm}) .^{40}$, 
${ }^{41}$ The relative intensity of the single-particle peak increases faster for the PEG sample, indicating the effect of the modifier in producing longer wires. This demonstrates the ability to isolate the single-particle behavior within the composite. Through this mechanism, high surface area 3D foams of optically active foams can be applied hierarchically to complex 3D surfaces (Figure $6 \mathrm{c}$ and Supplementary Video 3). This is just a single example of functional particles that could be explored, and there are also other opportunities to create NW structures through other viscosity transitions. Overall, this study highlights that the formation of morphologies during ESD spray, despite being experimentally investigated for several decades, is still relatively under-investigated. The interplay of extreme electrostatic forces with phase separation and dynamic viscous fluidics can result in new and beautiful morphological evolution and create a novel manufacturing platform for functional materials and coatings.
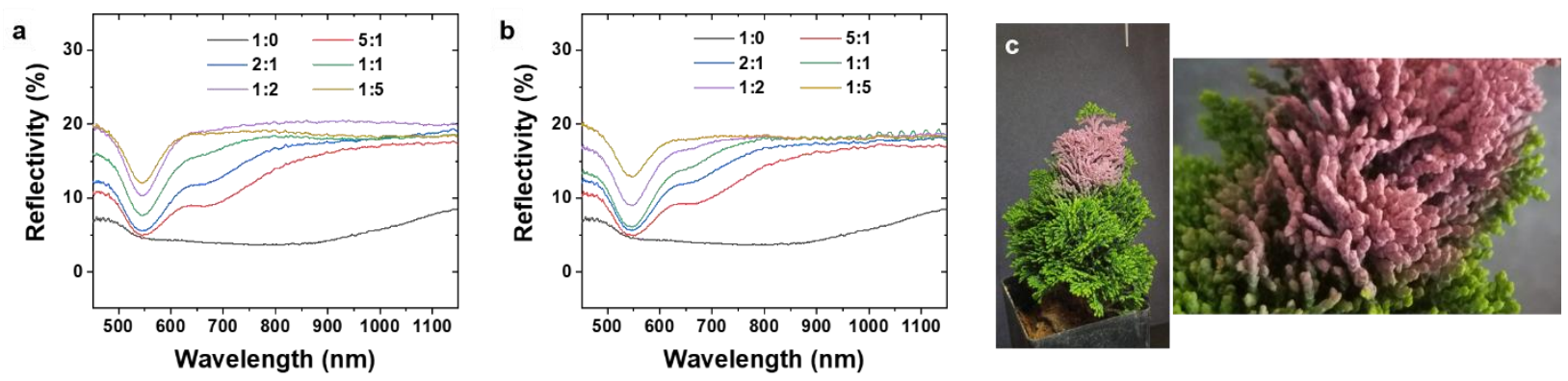

Figure 6: Blends of MC and gold nanoparticles. (a,b) Reflection spectra of (a) sprayed $0.3 \mathrm{wt} \% 14 \mathrm{kDa}$ MC:50 nm gold nanoparticles in different mass ratios; (b) sprayed 0.3 wt\% (5 14 kDa MC:1 PEG) : $50 \mathrm{~nm}$ gold nanoparticles in different mass ratios; All sprays were conducted at a spray distance of $4 \mathrm{~cm}$ with a flow rate of $0.15 \mathrm{~mL} / \mathrm{hr}$ for $30 \mathrm{~min}$ at the substrate temperature of $90{ }^{\circ} \mathrm{C}$ in $3: 2$ volume-basis water:ethanol blend. (c) Photographs of a complex 3D surface coating of a Thoweil Hinoki Cypress with 0.3 wt\% 5:1 (5 $14 \mathrm{kDa}$ MC:1 PEG) : $50 \mathrm{~nm}$ gold nanoparticles in 3:2 volume-basis water:ethanol blend at a minimum spray distance of $\sim 5 \mathrm{~cm}$ with a flow rate of $0.2 \mathrm{~mL} / \mathrm{hr}$ for $30 \mathrm{hr}$ at room temperature. The zoomed in view reveals the conformal coating of the forest on the tree.

\section{Acknowledgements}

J.P.S. acknowledges funding through the 3M Non-Tenured Faculty Award. X. Y. acknowledges funding through the American Chemical Society Petroleum Research Fund No. 56884-DNI9.

\section{References}

1. Persano, L.; Camposeo, A.; Tekmen, C.; Pisignano, D. Macromolecular Materials and Engineering 2013, 298, (5), 504-520.

2. Blumberg, L. N.; Gursky, J. C.; Stein, P. C., Electrospraying of Thin Targets. Los Alamos Scientific Laboratory: 1962.

3. Yamashita, M.; Fenn, J. B. The Journal of Physical Chemistry 1984, 88, (20), 4451-4459.

4. Jaworek, A.; Sobczyk, A. T. J. Electrostatics 2008, 66, (3-4), 197-219.

5. Kavadiya, S.; Biswas, P. J. Aerosol Sci 2018.

6. Morota, K.; Matsumoto, H.; Mizukoshi, T.; Konosu, Y.; Minagawa, M.; Tanioka, A.; Yamagata, Y.; Inoue, K. Journal of colloid and interface science 2004, 279, (2), 484-492.

7. Mizukoshi, T.; Matsumoto, H.; Minagawa, M.; Tanioka, A. Journal of applied polymer science 2007, 103, (6), 3811-3817. 
8. Nasir, M.; Matsumoto, H.; Minagawa, M.; Tanioka, A.; Danno, T.; Horibe, H. Polymer journal 2009, 41, (5), 402-406.

9. Almería, B.; Gomez, A. Journal of colloid and interface science 2014, 417, 121-130.

10. Altmann, K.; Schulze, R.-D.; Friedrich, J. Thin solid films 2014, 564, 269-276.

11. Rietveld, I. B.; Kobayashi, K.; Yamada, H.; Matsushige, K. Journal of colloid and interface science 2006, 298, (2), 639-651.

12. Kawakami, K. International journal of pharmaceutics 2012, 433, (1-2), 71-78.

13. Rietveld, I. B.; Kobayashi, K.; Yamada, H.; Matsushige, K. Soft Matter 2009, 5, (3), 593-598.

14. Rezvanpour, A.; Wang, C.-H. Chemical engineering science 2011, 66, (17), 3836-3849.

15. Hao, S.; Wang, Y.; Wang, B.; Deng, J.; Zhu, L.; Cao, Y. Materials Science and Engineering: C 2014, 39, 113-119.

16. Xie, J.; Tan, J. C.; Wang, C.-H. Journal of pharmaceutical sciences 2008, 97, (8), 3109-3122.

17. Hoburg, J. F. ITIA 1982, (6), 666-672.

18. Han, Y.; Dong, J. Journal of Micro and Nano-Manufacturing 2018, 6, (4).

19. Almería, B.; Gomez, A. J. Colloid Interface Sci. 2014, 417, 121-130.

20. Gomez, A.; Tang, K. Physics of Fluids 1994, 6, (1), 404-414.

21. Collins, R. T.; Sambath, K.; Harris, M. T.; Basaran, O. A. Proceedings of the National Academy of Sciences 2013, 110, (13), 4905-4910.

22. Duft, D.; Achtzehn, T.; Müller, R.; Huber, B. A.; Leisner, T. Nature 2003, 421, (6919), 128-128.

23. Merrill, M. H.; Pogue, I. I. I. W. R.; Baucom, J. N. Journal of Micro and Nano-Manufacturing 2015, 3, (1), 011003-011003.

24. Zhao, X.-Y.; Johnston, D. E.; Rodriguez, J. C.; Tao, Z.; Mi, B.-X.; Deng, W. Macromolecular Materials and Engineering 2017, 302, (9), 1700090.

25. Sirignano, W. A., Fluid dynamics and transport of droplets and sprays. Cambridge university press: 2010.

26. McAllister, J. W.; Schmidt, P. W.; Dorfman, K. D.; Lodge, T. P.; Bates, F. S. Macromolecules 2015, 48, (19), 7205-7215.

27. Morozova, S. Polym. Int. 2020.

28. Lott, J. R.; McAllister, J. W.; Wasbrough, M.; Sammler, R. L.; Bates, F. S.; Lodge, T. P.

Macromolecules 2013, 46, (24), 9760-9771.

29. McAllister, J. W.; Lott, J. R.; Schmidt, P. W.; Sammler, R. L.; Bates, F. S.; Lodge, T. P. ACS Macro Letters 2015, 4, (5), 538-542.

30. Morozova, S.; Schmidt, P. W.; Metaxas, A.; Bates, F. S.; Lodge, T. P.; Dutcher, C. S. ACS Macro Letters 2018, 7, (3), 347-352.

31. Takeshita, H.; Saito, K.; Miya, M.; Takenaka, K.; Shiomi, T. Journal of Polymer Science Part B: Polymer Physics 2010, 48, (2), 168-174.

32. Levy, G.; Schwarz, T. W. Journal of the American Pharmaceutical Association 1958, 47, (1), 44-46.

33. Xu, Y.; Li, L.; Zheng, P.; Lam, Y. C.; Hu, X. Langmuir 2004, 20, (15), 6134-6138.

34. Salehi, M. B.; Sefti, M. V.; Moghadam, A. M.; Koohi, A. D. Journal of Macromolecular Science, Part B 2012, 51, (3), 438-451.

35. Frenot, A.; Henriksson, M. W.; Walkenström, P. J. Appl. Polym. Sci. 2007, 103, (3), 1473-1482.

36. Gañán-Calvo, A. M.; Dávila, J.; Barrero, A. J. Aerosol Sci 1997, 28, (2), 249-275.

37. Nasatto, P. L.; Pignon, F.; Silveira, J. L.; Duarte, M. E. R.; Noseda, M. D.; Rinaudo, M. Polymers 2015, 7, (5), 777-803.

38. Lei, L.; Kovacevich, D. A.; Nitzsche, M. P.; Ryu, J.; Al-Marzoki, K.; Rodriguez, G.; Klein, L. C.; Jitianu, A.; Singer, J. P. ACS Applied Materials \& Interfaces 2018, 10, (13), 11175-11188.

39. Kovacevich, D. A.; Lei, L.; Han, D.; Kuznetsova, C.; Lee, H.; Singer, J. P. ChemRxiv 2019.

40. Wei, Q. H.; Su, K. H.; Durant, S.; Zhang, X. Nano Letters 2004, 4, (6), 1067-1071.

41. Su, K. H.; Wei, Q. H.; Zhang, X.; Mock, J. J.; Smith, D. R.; Schultz, S. Nano Letters 2003, 3, (8), 1087-1090. 
